Development and experimental assessment of a numerical modelling code to aid the design of profile extrusion cooling tools

O. S. Carneiro, A. Rajkumar, C. Fernandes, L. L. Ferrás, F. Habla, and J. M. Nóbrega

Citation: AIP Conference Proceedings 1896, 120008 (2017); doi: 10.1063/1.5008146

View online: http://dx.doi.org/10.1063/1.5008146

View Table of Contents: http://aip.scitation.org/toc/apc/1896/1

Published by the American Institute of Physics 


\title{
Development and Experimental Assessment of a Numerical Modelling Code to Aid the Design of Profile Extrusion Cooling Tools
}

\author{
O.S. Carneiro ${ }^{1, \mathrm{a})}$, A. Rajkumar ${ }^{1, \mathrm{~b})}$, C. Fernandes ${ }^{1, \mathrm{c})}$, L.L. Ferrás ${ }^{1, \mathrm{~d})}$, F. Habla ${ }^{2, \mathrm{e})}$, \\ J.M. Nóbrega ${ }^{1, f)}$ \\ ${ }^{1}$ IPC/i3N-Institute for Polymers and Composites, Department of Polymer Engineering, University of Minho, \\ Guimarães, Portugal \\ ${ }^{2}$ Technische Universitat Munchen, Catalysis Research Center and Chemistry \\ Department, Munchen, Germany

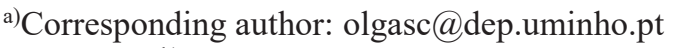 \\ b)rajkananth@gmail.com \\ c)cbpf@dep.uminho.pt \\ d)luis.ferras@dep.uminho.pt \\ e)florian.habla@ch.tum.de \\ f)mnobrega@dep.uminho.pt
}

\begin{abstract}
On the extrusion of thermoplastic profiles, upon the forming stage that takes place in the extrusion die, the profile must be cooled in a metallic calibrator. This stage must be done at a high rate, to assure increased productivity, but avoiding the development of high temperature gradients, in order to minimize the level of induced thermal residual stresses. In this work, we present a new coupled numerical solver, developed in the framework of the OpenFOAM ${ }^{\circ}$ computational library, that computes the temperature distribution in both domains simultaneously (metallic calibrator and plastic profile), whose implementation aimed the minimization of the computational time. The new solver was experimentally assessed with an industrial case study.
\end{abstract}

\section{INTRODUCTION}

The extrusion of thermoplastic profiles employs an extrusion line encompassing an extruder coupled to an extrusion die that shapes the polymer melt into the desired geometry, a metallic calibrator, refrigerated by cold water circulating in cooling channels, where the profile is cooled down, and a pulling unit that imposes the profile linear speed, i.e. the production rate. The cooling process should be performed at a high rate, to assure increased productivity, but avoiding the development of high temperature gradients, in order to minimize the level of induced thermal residual stresses. These objectives are conflicting, since an increase of the cooling rate usually induces higher temperature gradients [1], and vice-versa. Due to the non-linear nature of the thermoplastic material behaviour and the large number of processing variables involved, the employment of numerical modelling tools is mandatory to properly understand the cooling system behaviour, and to guide its design. To model the profile extrusion cooling system (calibrator), the temperature distribution must be computed both at the metallic calibrator and at the polymeric profile, which are in contact through a common interface. The traditional computational tools available for this purpose, follow an explicit approach that starts by assuming a certain temperature distribution in both domains, which are used to compute the interface heat fluxes. Subsequently, these new heat fluxes are used to update again the temperature distribution in both domains. This gives rise to an intensive iterative process, which usually must be substantially relaxed to assure convergence. This relaxation has a direct impact on the computational time consumed.

In this work, we present the development of a new coupled numerical solver [2], implemented in the framework of the OpenFOAM ${ }^{\circledR}$ computational library, that computes the temperature distribution in both domains simultaneously, in an implicit way, aiming to minimize the computational time. Contact resistance at the polymercalibrator interface is assumed [3] and the heat transfer process is modelled using the volume averaging [4-6] approach, which allows devising a single governing equation valid in both domains. Unstructured meshes [7] were used, in order to enable the use of the solver to model the cooling process of complex geometry plastics profiles and

Proceedings of the 20th International ESAFORM Conference on Material Forming AIP Conf. Proc. 1896, 120008-1-120008-6; https://doi.org/10.1063/1.5008146 Published by AIP Publishing. 978-0-7354-1580-5/\$30.00 
to accurately tackle curved geometries, such as, for example, those limiting the circular calibrator cooling channels. The solver was verified by comparison of its predictions with analytical solutions (for simple problems), considering both perfect contact and heat contact resistance at the polymer-calibrator interface, through the Method of Manufactured Solutions (MMS) and with results published in the literature. The details of the verification studies performed can be found elsewhere [2]. In this work, the new solver is experimentally assessed with an industrial driven case study, considering a swimming pool cover profile, cooled by three calibrators in series.

\section{SOLVER DEVELOPMENT}

Eq. 1 is the simplified energy conservation equation corresponding to the problem under consideration, which comprises the relevant terms,

$$
\frac{\partial\left(\rho c_{P} T\right)}{\partial t}+\nabla \cdot\left(\mathbf{U} \rho c_{P} T\right)-\nabla \cdot k \nabla T=0
$$

where $T$ is the temperature, $t$ is the time, $\rho$ is the density, $c_{P}$ is the heat capacity, $\mathbf{U}$ is the velocity vector and $k$ is the thermal conductivity. This equation can be solved numerically if adequate boundary conditions are defined at the system boundaries and at the polymer-calibrator interface. In case of perfect contact the temperature $T$ and the interface heat flux $\dot{\mathbf{q}}$ would be continuous, and given by:

$$
\begin{gathered}
T_{\mid \text {interface, } p}-T_{\mid \text {interface, } c}=0 \\
\left(\dot{\mathbf{q}}_{p}-\dot{\mathbf{q}}_{c}\right) \cdot \mathbf{n}=\left(k_{p} \nabla T_{\mid \text {interface }, p}-k_{c} \nabla T_{\mid \text {interface, },}\right) \cdot \mathbf{n}=0
\end{gathered}
$$

where $\mathbf{n}$ denotes the interface normal vector pointing from the profile (subscript $\mathrm{p}$ ) to the calibrator (subscript $\mathrm{c}$ ). However, under real processing conditions there is a contact resistance at the polymer-calibrator interface, which results in a temperature discontinuity between both domains [2]. Thus Eq. (2) should be replaced by:

$$
T_{\mid \text {interface, } p}-T_{\mid \text {interface, } c}=\frac{\dot{\mathbf{q}}_{c} \cdot \mathbf{n}}{h}
$$

where $h$ denotes the interface heat transfer coefficient.

The technique of conditional volume averaging [4-6] was used to devise an equation for the mixture temperature $T_{m}$ that fulfills the Eqs. (3) and (4), i.e. the more realistic case. Using the proposed methodology [4-6] Eq. (1) is first conditioned and then volume averaged, which leads to,

$$
\frac{\partial\left(\alpha_{i} \bar{\rho}^{i} \bar{c}_{P}^{i} \bar{T}^{i}\right)}{\partial t}+\nabla \cdot\left(\alpha_{i} \overline{\mathrm{U}}^{i} \bar{\rho}^{i} \bar{c}_{P}^{i} \bar{T}^{i}\right)=\nabla \cdot \alpha_{i} \bar{k}^{i} \nabla \bar{T}^{i}-\overbrace{k \nabla T \cdot \mathrm{n}}^{\text {Interfacial }} \sum
$$

where $\alpha_{i}$ is the volume fraction of each of the phases (domains) $i=p, c$. The last term on the r.h.s. is the interfacial source [5] and the overbar denotes the average value inside the volume. Summing up Eq. (5) for both phases and using mixture quantities $[4,5]$ we obtain,

$$
\frac{\partial\left(\rho_{m} c_{P, m} T_{m}\right)}{\partial t}+\nabla \cdot\left(\mathbf{U}_{m} \rho_{m} c_{P, m} T_{m}\right)=\nabla \cdot \alpha_{p} \bar{k}^{p} \nabla \bar{T}^{p}+\nabla \cdot \alpha_{c} \bar{k}^{c} \nabla \bar{T}^{c}
$$

Note that the interfacial source terms sum up to zero for phases $p$ and $c$ due to continuity of the heat flux. Since the only dependent variable will be $T_{m}$ we need to find expressions for the two diffusion terms on the r.h.s. of Eq. (6). Introducing the mixture diffusion term: 


$$
\begin{aligned}
\nabla \cdot k_{m} \nabla T_{m}= & \nabla \cdot\left[\left(\alpha_{p} \bar{k}^{p}+\alpha_{c} \bar{k}^{c}\right) \nabla\left(\alpha_{p} \bar{T}^{p}+\alpha_{c} \bar{T}^{c}\right)\right] \\
= & \nabla \cdot\left[\bar{k}^{p} \nabla \alpha_{p} \bar{T}^{p}+\bar{k}^{c} \nabla \alpha_{c} \bar{T}^{c}+\alpha_{p} \bar{k}^{p} \nabla \alpha_{p} \bar{T}^{p}+\alpha_{c} \bar{k}^{c} \nabla \alpha_{c} \bar{T}^{c}\right. \\
& \left.-\alpha_{c} \bar{k}^{p} \nabla \alpha_{p} \bar{T}^{p}-\alpha_{p} \bar{k}^{c} \nabla \alpha_{c} \bar{T}^{c}\right]
\end{aligned}
$$

then subtracting Eq. (7) to Eq. (6), and using the identity $\nabla \alpha_{c}=-\nabla \alpha_{p}$, one obtains,

$$
\begin{gathered}
\frac{\partial\left(\rho_{m} c_{P, m} T_{m}\right)}{\partial t}+\nabla \cdot\left(\mathbf{U}_{m} \rho_{m} c_{P, m} T_{m}\right)-\nabla \cdot k_{m} \nabla T_{m}= \\
-\nabla \cdot\left[\left(\alpha_{p} \bar{k}^{p}+\alpha_{c} \bar{k}^{c}\right)\left(\bar{T}^{p}-\bar{T}^{c}\right) \nabla \alpha_{p}-\alpha_{p} \alpha_{c}\left(\bar{k}^{p}-\bar{k}^{c}\right)\left(\nabla \bar{T}^{p}-\nabla \bar{T}^{c}\right)\right]
\end{gathered}
$$

The term $\bar{T}^{p}-\bar{T}^{c}$ can be calculated using Eq. (4), because $\nabla \alpha_{p}$ is non-zero only at the polymer-calibrator interface, and the term $\nabla \bar{T}^{p}-\nabla \bar{T}^{c}$ should be computed using the assumption that the temperature gradient is normal to the profile-calibrator interface, i.e., $\nabla \bar{T}^{p}-\nabla \bar{T}^{c} \approx\left(\nabla \bar{T}^{p} \cdot \mathbf{n}-\nabla \bar{T}^{c} \cdot \mathbf{n}\right) \mathbf{n}$. Hence, Eq. (3) is used to express $\nabla \bar{T}^{c} \cdot \mathbf{n}$ in terms of $\nabla \bar{T}^{p} \cdot \mathbf{n}$ leading to,

$$
\begin{gathered}
\frac{\partial\left(\rho_{m} c_{P, m} T_{m}\right)}{\partial t}+\nabla \cdot\left(\mathbf{U}_{m} \rho_{m} c_{P, m} T_{m}\right)-\nabla \cdot k_{m} \nabla T_{m}= \\
-\nabla \cdot\left[\left(\left(\alpha_{p} \bar{k}^{p}+\alpha_{c} \bar{k}^{c}\right) \frac{\bar{k}^{c}}{h} \nabla \alpha_{p}-\alpha_{p} \alpha_{c}\left(\bar{k}^{p}-\bar{k}^{c}\right)\left(1-\frac{\bar{k}^{p}}{\bar{k}^{c}}\right) \mathbf{n}\right) \nabla \bar{T}^{p} \cdot \mathbf{n}\right]
\end{gathered}
$$

The last step of the formulation is to obtain an expression for $\nabla \bar{T}^{p} \cdot \mathbf{n}$. For that, the gradient of the mixture temperature $T_{m}$ is calculated by $\nabla T_{m}=\alpha_{p} \nabla \bar{T}^{p}+\alpha_{c} \nabla \bar{T}^{c}+\left(\bar{T}^{p}-\bar{T}^{c}\right) \nabla \alpha_{p}$ and scalar product with $\mathbf{n}$ results in $\nabla \bar{T}^{p} \cdot \mathbf{n}=\left(\nabla T_{m} \cdot \mathbf{n}\right) /\left(\alpha_{p}+\alpha_{c} \frac{\bar{k}^{p}}{\bar{k}^{c}}+\frac{\bar{k}^{p}}{h} \nabla \alpha_{p} \cdot \mathbf{n}\right)$. This yields the final equation for the mixture temperature $T_{m}$,

$$
\frac{\partial\left(\rho_{m} c_{P, m} T_{m}\right)}{\partial t}+\nabla \cdot\left(\mathbf{U}_{m} \rho_{m} c_{P, m} T_{m}\right)-\nabla \cdot k_{m} \nabla T_{m}=-\nabla \cdot\left[\frac{k_{m} \nabla \alpha_{p}-\alpha_{p} \alpha_{c}\left(\bar{k}^{p}-\bar{k}^{c}\right)\left(\frac{1}{\bar{k}^{p}}-\frac{1}{\bar{k}^{c}}\right) h \mathbf{n}}{h\left(\frac{\alpha_{p}}{\bar{k}^{p}}+\frac{\alpha_{c}}{\bar{k}^{c}}\right)+\nabla \alpha_{p} \cdot \mathbf{n}} \nabla T_{m} \cdot \mathbf{n}\right]
$$

This last equation is numerically solved in the open-source computational library OpenFOAM $₫$, to compute the full system temperature distribution. The software is based on the Finite Volume Method and is capable of handling arbitrary unstructured three-dimensional meshes. For discretization purposes, the interpolation of the dependent variable $T_{m}$ onto the cell-faces for calculation of the convective term is done with the third-order QUICK scheme. Based on numerical tests it was found that a generalized geometric-algebraic multi-grid (GAMG) solver in conjunction with a diagonal incomplete-Cholesky smoother, with a tolerance of $10^{-7}$, yields the fastest convergence rates.

\section{INDUSTRIAL DRIVEN CASE STUDY}

The new solver was tested modeling the cooling stage taking place in an existing industrial extrusion line for the production of a polycarbonate (PC) swimming pool cover profile. The cross-section geometry of the PC profile is illustrated in Fig. 1(a) and the layout of the cooling system, comprising three stainless steel calibrators in series, is shown in Fig. 1(c). The location of the main extrusion line components, and length and temperature of each calibrator are defined in Fig. 2. The calibrators cooling channels have a zigzag layout (see Fig. 1(c)) and their diameter was 6.75 
$\mathrm{mm}$. The extrusion temperature was $228^{\circ} \mathrm{C}$, the room temperature $18^{\circ} \mathrm{C}$, and the pulling system speed was set to 1.28 $\mathrm{m} / \mathrm{min}$. The boundary conditions and parameters used in the simulation are shown in Fig. 3 and Table 1, respectively.

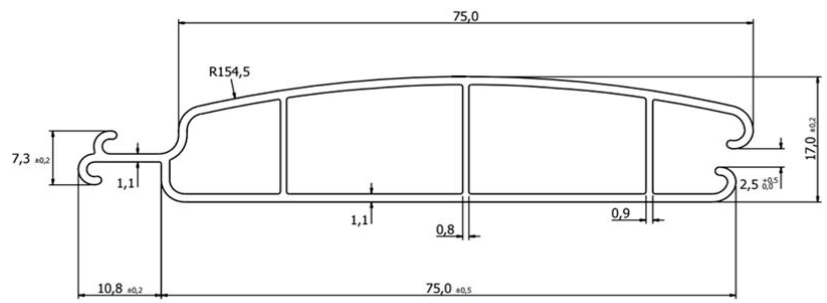

(a)

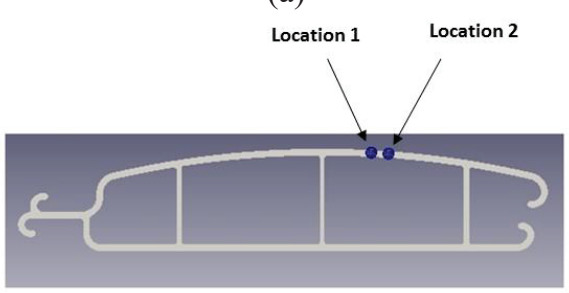

(b)

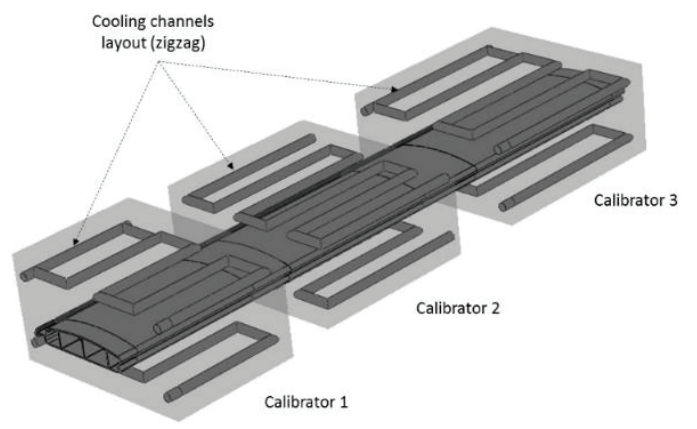

(c)

FIGURE 1. Complex experimental case-study (swimming pool cover) used in the solver assessment: (a) geometry of the plastic profile (dimensions in $\mathrm{mm}$ ); (b) limits of the zone where the temperatures were compared for assessment purposes; (c) layout of the calibration/cooling system employed.

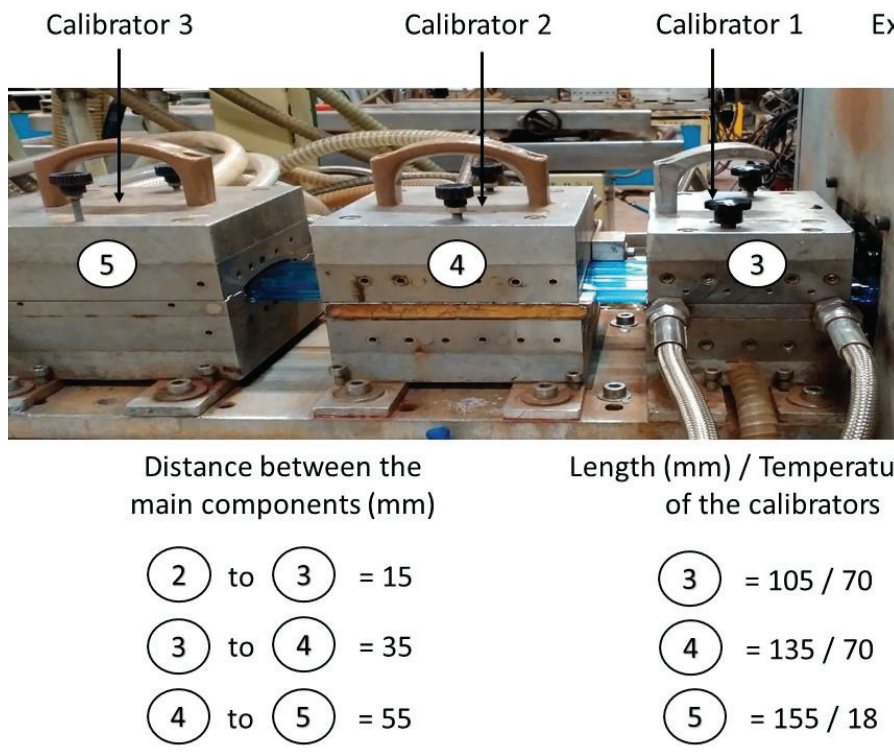

FIGURE 2. Main components of the extrusion line and distance between them; length and temperature of each calibrator.

The mesh used in the simulation was selected after performing a refinement sensitivity study, and comprises more than 4 million of computational cells. An example of the mesh used, corresponding to a cross section of the domain where the plastic profile and the metallic calibrator exist, is illustrated in Fig. 4.

Experimental temperature measurements were taken at the profile top surface, along its length, using an IR thermographic camera, in the vicinity of the locations indicated in Fig. 1(b). Due to the practical difficulties in measuring the temperature at a precise location, the experimental data collected in that zone were averaged and 
compared with the average temperature values numerically computed between the two fixed limiting locations shown in Fig. 1(b).

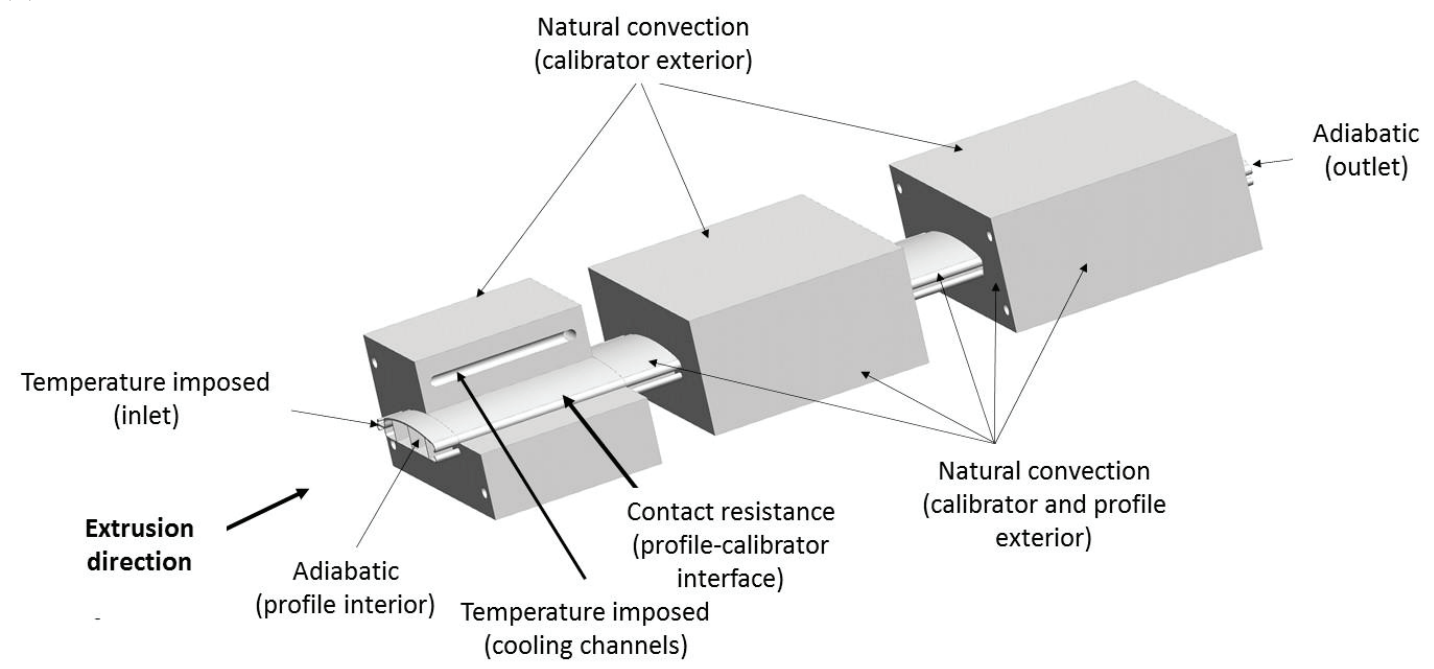

FIGURE 3. Boundary conditions used in the numerical simulation.

TABLE 1. Materials' properties and heat transfer coefficients used in the numerical simulation.

\begin{tabular}{cc}
\hline Parameter & Value \\
\hline Polycarbonate properties: $k_{p}(\mathrm{~W} / \mathrm{mK}) / c_{p}(\mathrm{~J} / \mathrm{kgK}) / \rho_{p}\left(\mathrm{~kg} / \mathrm{m}^{3}\right)$ & $0.255 / 1808 / 1090$ \\
Stainless steel properties: $k_{c}(\mathrm{~W} / \mathrm{mK}) / c_{c}(\mathrm{~J} / \mathrm{kgK}) / \rho_{c}\left(\mathrm{~kg} / \mathrm{m}^{3}\right)$ & $28.5 / 510 / 7740$ \\
Air heat transfer coefficient (free convection) $\left(\mathrm{W} / \mathrm{m}^{2} \mathrm{~K}\right)$ & 5 \\
Profile-calibrator interface heat transfer coefficient $\left(\mathrm{W} / \mathrm{m}^{2} \mathrm{~K}\right)$ & 500 \\
\hline
\end{tabular}

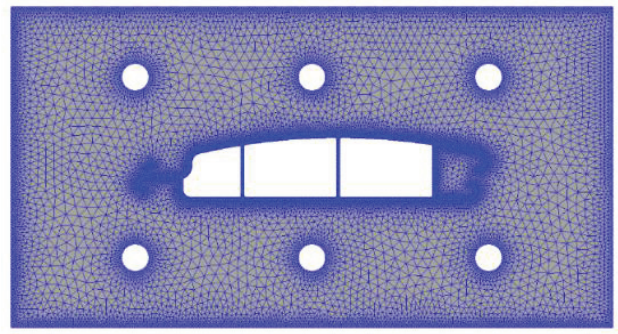

FIGURE 4. Typical mesh used in the numerical simulation, corresponding to plastic and calibrator domains.

The results computed and the experimental measurements are shown in Fig. 5. As illustrated, the temperatures at the cooling system outlet are almost superimposed and the evolution of the temperature along the cooling system is adequately predicted. In fact, the solver is capable to predict a decreasing temperature for the profile surface, along each calibrator, and also the progressive decrease in efficiency along these cooling units length, due to the progressive decrease of the profile surface temperature. Also, an increase in the profile surface temperature is predicted in the annealing zones (between calibrators), due to the heat conducted from the hotter inner profile zones. The differences between predicted and experimental values are very low, of the order of $1-2 \%$, with the exception of the points located in the first annealing zone (between calibrators 1 and 2), where a maximum difference of $8 \%$ was observed. Having 
in mind the practical difficulties in measuring temperatures at accurate locations, we can conclude that the numerical code is capable to model the behavior of this type of systems, being the predictions physically sound and realistic.

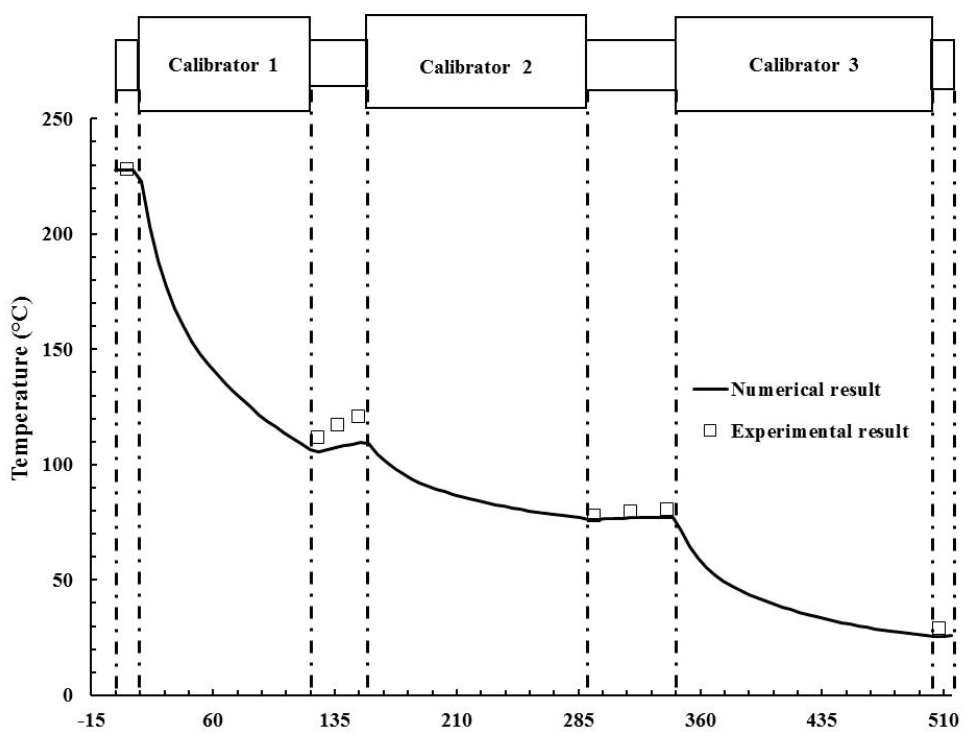

FIGURE 5. Evolution of the average of the temperatures computed at two locations (See Fig 1(a)) of the top profile surface and corresponding values experimentally measured.

\section{CONCLUSIONS}

The main conclusions of this work are the following: i) the new solver is able to compute the temperature distribution in a two domain system and to account for a discontinuity at the interface; ii) the simulation results provided for an industrial complex case are very close to those obtained experimentally.

\section{ACKNOWLEDGEMENTS}

The authors would like to thank the financial funding by FEDER through the COMPETE 2020 Program, the National Funds through FCT (Portuguese Foundation for Science and Technology) under the project UID/CTM/50025/2013 and the Search-ON2 HPC infrastructure of UMinho (NORTE-07-0162-FEDER-000086), cofunded by the North Portugal Regional Operational Programme (ON.2-O Novo Norte), under the NSRF through the ERDF. A. Rajkumar acknowledges the MIT-Portugal program and FCT for the funding through the scholarship $(\mathrm{SFRH} / \mathrm{BD} / 51943 / 2012)$ and L.L. Ferrás the financial support by FCT through the scholarship SFRH/BPD/100353/2014.

\section{REFERENCES}

1. J. M. Nóbrega, O. S. Carneiro, J. A. Covas, F. T. Pinho and P. J. Oliveira, Polym. Eng. Sci. 44, 2216-2228 (2004).

2. F. Habla, C. Fernandes, M. Maier, L. Densky, L. Ferrás, O. S. Carneiro, O. Hinrichsen and J. M. Nóbrega, Appl. Thermal Eng. 100, 538-552 (2016).

3. O. S. Carneiro, J. M. Nóbrega, A. Mota and C. Silva, Polym. Testing 32, 1154-1161 (2013).

4. C. Dopazo, Journal of Fluid Mechanics 81, 433-438 (1977).

5. H. Marschall, K. Hinterberger, C. Schüler, F. Habla and O. Hinrichsen, Chemical Engineering Science 78, 111127 (2012).

6. F. Habla, L. Dietsche and O. Hinrichsen, AIChE Journal 59, 3914-3927 (2013).

7. J. H. Ferziger and M. Peric, Computational Methods for Fluid Dynamics, $3^{\text {rd }}$ Ed. (Springer, 2012), pp. 26. 